\title{
Trustworthiness Management in Sharing CDN Infrastructure
}

\author{
Tai-Won Um \\ Dept. of Information and \\ Communication Engineering, \\ Chosun University, Gwangju, 61452, \\ Korea (Rep. of) \\ twum@chosun.ac.kr
}

\author{
Gyu Myoung Lee \\ Dept. of Computer Science, \\ Liverpool John Moores University, \\ Liverpool, L3 3AF, UK. \\ g.m.lee@ljmu.ac.uk
}

\author{
Hyun-Woo Lee \\ Electronics and Telecommunications \\ Research Institute, Daejeon 34129, \\ Korea (Rep. of) \\ hwlee@etri.re.kr
}

\begin{abstract}
Sharing Content Delivery Network (CDN) technologies such as CDN interconnection and cloud-based $\mathrm{CDN}$ have facilitated access to the huge volume of content in a cost-effective way. However, content distribution through shared CDN nodes is vulnerable to a wide variety of uncertainties, including unexpected node failure, intentional node disruption for maintenance or potential discrimination of content based on ownership. Trustworthiness can be considered as a key property to overcome the perception of uncertainty before delivering content through sharing CDN infrastructure and provide reliable broadcasting and telecommunications services. Establishing trustworthiness in sharing CDN infrastructure is a challenging task in the absence of the referenced framework. Therefore, we propose a trustworthiness management framework for sharing CDN infrastructure.
\end{abstract}

Keywords-Trust, trustworthiness management, trust information, sharing $C D N$

\section{INTRODUCTION}

Rapidly growing media traffic offers challenges to internet service providers as well as video content providers [1]. A content delivery network (CDN) is a prevalently used media distribution system, where content delivery components are interconnected and transmit data from broadcasting and telecommunications systems. It can provide high-quality and scalable media services, while reducing service response time and network congestion [2]. All these advantages make more and more service providers to adopt CDNs for their content distribution [3].

Most of media services including video on demand (VoD) and real-time streaming need not only sustainable bandwidth but also reliability with a certain level of quality. Furthermore, new media services, such as interactive multimedia, cloudbased gaming services, require strict quality of service (QoS) constraints.

Recently, sharing CDN technologies such as cloud-based $\mathrm{CDN}$ and CDN interconnection (CDNi) have been deployed in networks [4],[5]. These technologies basically borrow the resources needed to transfer data from cloud platforms or other CDN providers.

However, these sharing CDN schemes impose many threats ranging from unexpected node failure to intentional discrimination of content based on ownership. Trustworthiness can be considered as an important factor in maintaining sharing CDN eco-system process to overcome the perception of uncertainty before delivering content through shared CDN nodes. This paper proposes a trustworthiness management framework for sharing CDN infrastructure.

The remainder of this paper is organized as follows. We briefly review the related work in section II. Section III describes potential risks and necessity of trustworthiness in sharing CDN infrastructure and the proposed trustworthiness management framework is provided in Section IV. Finally, section $\mathrm{V}$ gives some concluding remarks.

\section{RELATED WORK}

\section{A. Sharing CDN Infrastructure}

A cloud computing is a model for enabling ubiquitous access to shared resources and dynamically assigning resources according to demand [4]. Adopting and utilizing this cloud computing, CDN providers can build their services efficiently and cost-effectively by renting the cloud resources.

The basic framework to dynamically construct the cloudbased CDN was proposed in [2], wherein replica servers used to store copies of original content are virtually created and modified on the cloud infrastructure. P. Frangoudis presented the design of a content-delivery-network-as-a-service architecture, which enables a telecom operator to open up its cloud infrastructure for content providers [6].

One the other hand, the CDN service coverage is typically regionally bound because its access users can receive contents from subscribed CDNs. In order to increase the benefit of CDN services, CDN providers should extend local CDN service coverage across the network domains [5]. CDNi is a method interconnecting two independent CDNs that enables one to deliver content on behalf of the other. 


\section{B. Trustworthiness Management}

Trustworthiness management has been highlighted as a key issue in the mediation and handling of commercial services, as well as the decision making in business processes.

To build converged ICT services and a reliable information infrastructure, ITU-T SG13 has been working on future trusted information and communication technology (ICT) infrastructures and recently published the recommendation Y.3052 [7] regarding the concept of trust, a trust relationship model and trust evaluation. According to the Y.3052, trust is defined as "the measurable belief and/or confidence which represents accumulated value from history and the expecting value for the future", and trust indicators are used to calculate a trust index by combining qualitative and quantitative attributes of trust.

Our previous work [8] presented a trust evaluation model called REK, comprised of the triad of trust indicators: Reputation, Experience and Knowledge as shown in TABLE I. The REK model covers multi-dimensional aspects of trust by incorporating heterogeneous information from personal experiences to global opinions.

TABLE I. THREE INDICATORS IN THE REK TRUST EVALUATION MODEL

\begin{tabular}{c||c|c|c}
\hline \multicolumn{1}{c|}{ Indicator } & Reputation & Experience & Knowledge \\
\hline $\begin{array}{c}\text { Main sources } \\
\text { of information }\end{array}$ & $\begin{array}{c}\text { public evidence } \\
\text { on a trustee }\end{array}$ & $\begin{array}{c}\text { personal } \\
\text { expertise about } \\
\text { the situation and } \\
\text { the context }\end{array}$ & $\begin{array}{c}\text { understandings on } \\
\text { a trustee }\end{array}$ \\
\hline $\begin{array}{c}\text { trustworthiness } \\
\text { attribute }\end{array}$ & $\begin{array}{c}\text { indirect trustworthiness attributes } \\
\text { that are estimated from secondary } \\
\text { data }\end{array}$ & $\begin{array}{c}\text { direct } \\
\text { trustworthiness } \\
\text { attribute available } \\
\text { to a trustor at first } \\
\text { hand }\end{array}$ \\
\hline
\end{tabular}

\section{POTENTIAL RISKS AND NECESSITY OF TRUSTWORTHINESS IN SHARING CDN INFRASTRUCTURE}

The lack of trustworthiness is able to provoke various problems of the content delivery from content servers to a number of user devices passing through sharing CDN infrastructure. From the aspect of transport, all the entities in sharing CDN infrastructures could be exploited for malicious attacks, node failures, intentional node disruption for maintenance, and so on. From the aspect of service, many threats including the risks of the potential discrimination of content based on ownership could happen over other CDN provider's nodes.

In order to minimize unexpected risks and to maximize the survivability of sharing CDN-based services, trustworthiness management can be used to evaluate and verify that the functional components in sharing CDN infrastructures and services are working in expected ways. Within certain predictability and reliability, sharing CDN infrastructure could be operated in a trustworthy way. Furthermore, it will be robust to unexpected executions and failures of sharing CDN nodes.

Because sharing CDN infrastructure generally supports various media services and involves multiple stakeholders trustworthiness management is one of the most important functional capabilities in maintaining reliable CDN-based service and applications.

A trust index is quantitatively or qualitatively calculated and measured based on a trust evaluation model, and then used for the decision-making process not only by value-chains among multiple media stakeholders, but also by applications and service transactions in CDN environments.

Therefore, trustworthiness management capabilities need to be implemented in sharing CDN nodes, as well as CDN services and applications.

\section{TRUSTWORTHINESS MANAGEMENT FRAMEWORK OF SHARING CDN INFRASTRUCTURE}

The proposed architecture consists of a large number of diverse content delivery components as well as trustworthiness management components which interconnect with each other and cooperatively operate while satisfying a given trustworthy service level.

The basic procedure for building trustworthy content distribution paths is to collect trust related data from shared CDN nodes, calculate the trust index by using a trust evaluation model and make decision to select proper CDN nodes on the content distribution paths. Based on this procedure, the trustworthiness management framework to strengthen trustworthiness in sharing CDN infrastructure is presented in Fig. 1 for stakeholders in media ecosystem value chain. It consists of three major parts as follows:

\section{A. Trust Agent}

The trust agent (TA) is used to collect trust related data from the CDN capabilities with the following modules:

- TA interface is used to collect trust related data from various types of CDN service and delivery components.

- Trust data collection is responsible for gathering the data required to evaluate the trust level of CDN components.

- Trust data filtering is used to refine trust data sets for trust evaluation.

Various mechanisms can be used to extract trust related data depending on particular interactions among the CDN components, service nodes and user devices. For instance, trust data might be feedback from user devices to a CDN-based service node after each content delivery.

\section{B. Trust Information and Management System}

The trust information management system (TIMS) is used for modelling, reasoning, and managing trust data collected from TAs to check whether CDN components satisfy certain trust criteria.

- Trust Modelling is used to specify, annotate, and build trust relationships between CDN components for the 
purpose of reasoning trust data. By synthesizing trust indicators about reputation, experience and knowledge, REK trust model can consolidate the computational trust in the sharing CDN environment with high accuracy.

- Trust Evaluation is used to analyze and assess trust levels based on the trust model. There are various algorithms and mechanisms used for evaluating a trust index such as Graph-based algorithms, Machine Learning techniques, Natural Language Processing, etc.

- Trust Knowledge Gathering Interface is used to gather various types of factors related to trust, it is necessary to have an investigation on factors that can be collected and extracted from the environment.

\section{Trust Service Enabler}

The trust service enabler (TSE) is used to provide trust knowledge of CDN components for a service based on the sharing CDN infrastructure.

- Trust identity management (IdM) can be used to manage identification/authentication of CDN components.

- Trust-based recommendation provides recommendations to shared CDN components. A number of trustworthy components can be interconnected based on this trustbased recommendation information.

\section{CONCLUSION}

Building and validating trusted relationships in sharing CDN infrastructure will be a key factor to provide reliable and high-quality media services and applications. This paper focused on an architectural framework for trustworthiness management in sharing CDN infrastructure. First we identify several trust issues, which affects QoS constraints in existing CDN schemes. Then based on the ITU-T trust framework, we present trustworthiness management in order to overcome a wide variety of uncertainties in sharing CDN infrastructure.

\section{ACKNOWLEDGMENT}

This work was supported by Institute for Information \& communications Technology Promotion (IITP) grant funded by the Korea government (MSIT). [2015-0-00533, Development of TII(Trusted Information Infrastructure) S/W Framework for Realizing Trustworthy IoT Eco-system]

\section{REFERENCES}

[1] "Cisco Visual Networking Index: Forecast and Methodology, 20122017,” White paper, Cisco, May 29, 2013.

[2] A. Moreira et al., "A Case for Virtualization of Content Delivery Networks,” Proc. Winter Simulation Conf., Phoenix, AZ, USA, Dec. 1114, 2011, pp. 3178-3189.

[3] Z. Zhuang and C. Guo, "Building Cloud-Ready Video Transcoding System for Content Delivery Networks (CDNs)," Proc. IEEE, GLOBECOM, Anaheim, CA, USA, Dec. 3-7, 2012, pp. 2048-2053.

[4] E. Pacini et al., "SI-Based Scheduling of Scientific Experiments on Clouds,” IEEE Int. Conf. IDAACS, Berlin, Germany, vol. 02, Sept. 1214, 2013, pp. 699-704.

[5] Y. Bang et al., "CDN interconnection service trial: implementation and analysis,” IEEE Communications Magazine, vol. 54, iss. 6, Jun. 2016 , pp. 94.-100.

[6] P. Frangoudis et al., "CDN-As-a-Service Provision Over a Telecom Operator's Cloud," IEEE Transactions on Network and Service Management, vol. 14, iss. 3, Sep. 2017, pp. 702-716.

[7] ITU-T Recommendation Y.3052, “Overview of trust provisioning for information and communication technology infrastructures and services,” Mar. 2017.

[8] Nguyen Binh Truong et al, "Toward a Trust Evaluation Mechanism in the Social Internet of Things,” Sensors, Jun. 2017.

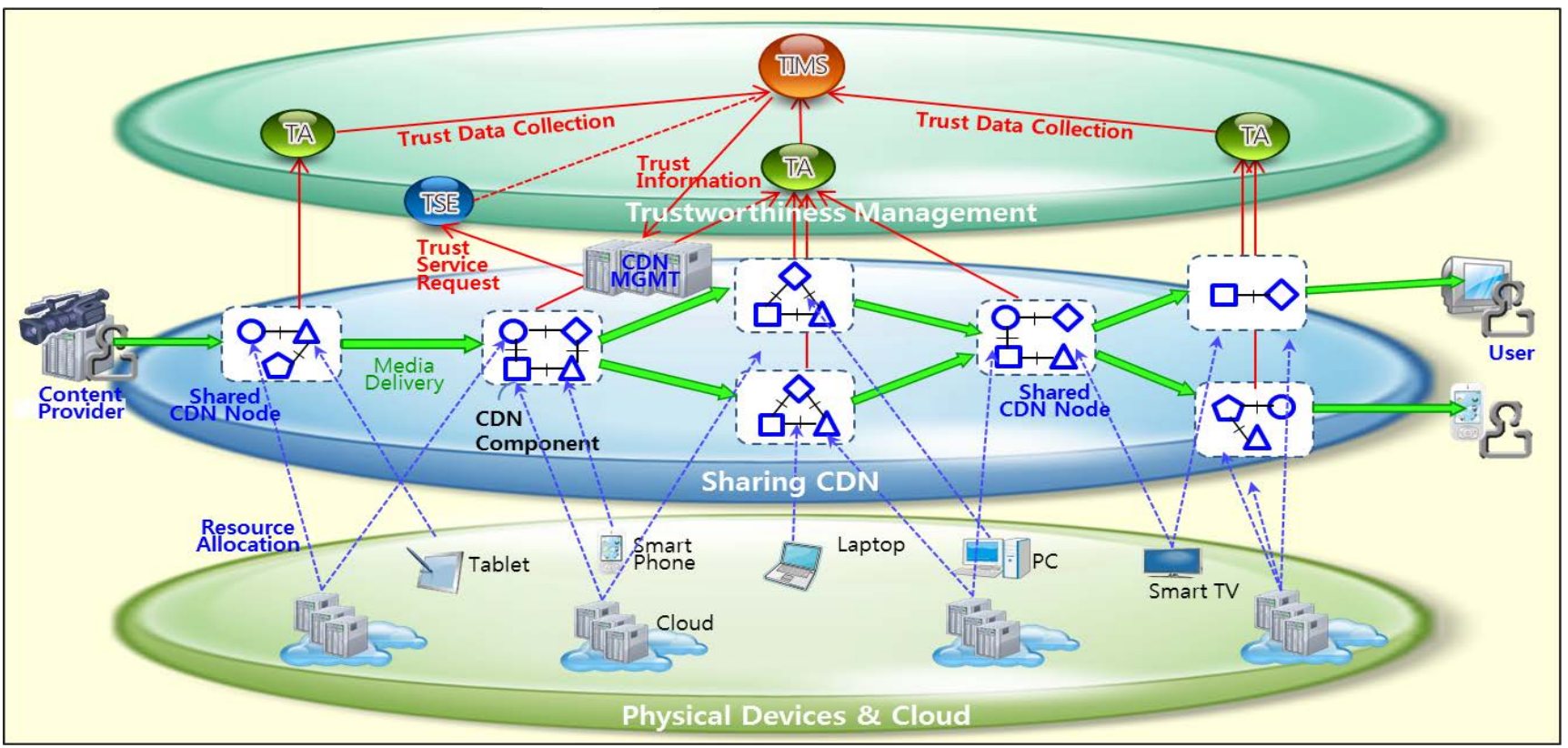

Fig. 1. Trustworthiness Management Framework for Sharing CDN Infrastructure 